\title{
Imaging Li-Ion Dynamics in Energy Storage Materials on the Nanometer Scales
}

\author{
N. Balke, ${ }^{*}$ A. Morozovska, ${ }^{* *}$ N. Dudney* and S.V. Kalinin* \\ * Oak Ridge National Laboratory, Oak Ridge, TN 37831 \\ ** Institute of Semiconductor Physics, National Academy of Science of Ukraine, 41, pr. Nauki, \\ $03028 \mathrm{Kiev}$, Ukraine
}

The electrochemical energy storage systems based on Li-based insertion and reconstitution chemistries are a vital aspect of energy technologies. Despite the wealth of device-level and atomistic studies, little is known on the mesoscopic mechanisms of ion diffusion and electronic transport on the level of grain clusters, individual grains, and extended defects.

The development of the capability for probing ion transport on the nanometer scale is a key to deciphering complex interplay between structure, functionality, and performance in these systems. Here we demonstrate how Scanning Probe Microscopy can be utilized to measure Li-ion mobility based on the strong strain-bias coupling in the system when local Li-ion concentrations are changed by electrical fields. Figure 1 illustrates the frequency dependence of the bias-induced strain for the Li-ion mapping in $\mathrm{LiCoO}_{2}$. The imaging capability, as well as time- and voltage spectroscopies analogous to traditional current based electrochemical characterization methods are developed. The reversible intercalation of Lithium and mapping electrochemical activity in $\mathrm{LiCoO}_{2}$ is demonstrated, illustrating higher Li-ion diffusivity at non-basal planes and grain boundaries. In Si anode device structures shown in Fig. 2, the direct mapping of Li-ion diffusion at extended defects and evolution of Li-ion diffusivity with charge state is explored, ultimately offering the sub-10 nm spatial resolution. The electrical field-dependence of Li-ion mobility is studied to determine the critical bias required for the onset of electrochemical transformation, potentially allowing reaction and diffusion processes in the battery system to be separated at each location. The Scanning Probe Microscopy measurements are compared with classical characterization methods such as cyclic voltammetry and electrochemical impedance spectroscopy. The prospects of Scanning Probe Microscopy for battery characterization are discussed.

\section{References}

[1] The research at ORNL was supported as part of the Fluid Interface Reactions, Structures and Transport (FIRST) Center, an Energy Frontier Research Center funded by the U.S. Department of Energy, Office of Science, Office of Basic Energy Sciences under Award Number ERKCC61. Part of worked is performed as a user proposal in the Center for Nanophase Materials Sciences at ORNL. 

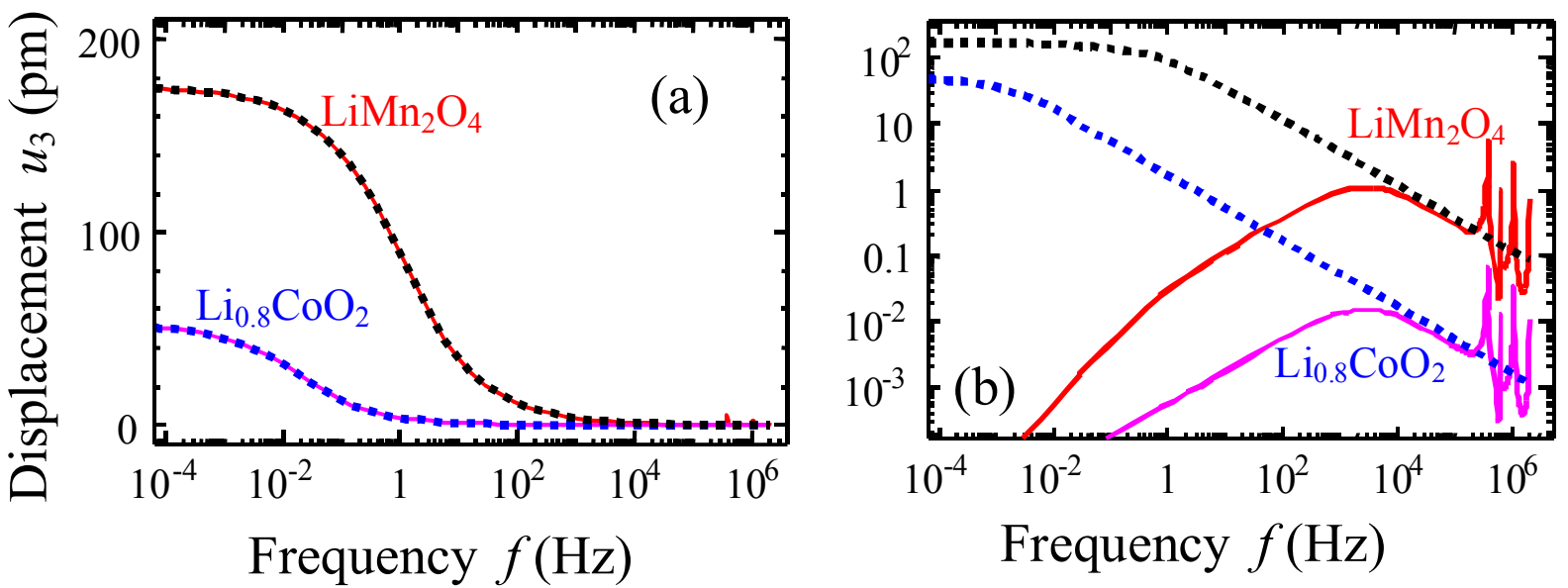

FIG. 1. (a) Absolute value of the material response (displacement $u_{3}(0, w)$, dotted curves); and the ESM response $\left(u_{3}(0, w)\right.$ multiplied by experimental transfer function, solid curves $)$ vs. driving frequency $f$ for determined concentration $\delta C / C_{\max }=0.05$ (i.e. $\lambda=0$ ) for $\mathrm{LiMn}_{2} \mathrm{O}_{4}$ and $\mathrm{Li}_{0.8} \mathrm{CoO}_{2}$ (labels near the curves). (d) Material response (dotted curves) and signal to noise ratio for ESM response divided by noise function $\sim\left(f_{0} / f+1\right)$ with $f_{0}=3 \mathrm{kHz}$ (solid curves).
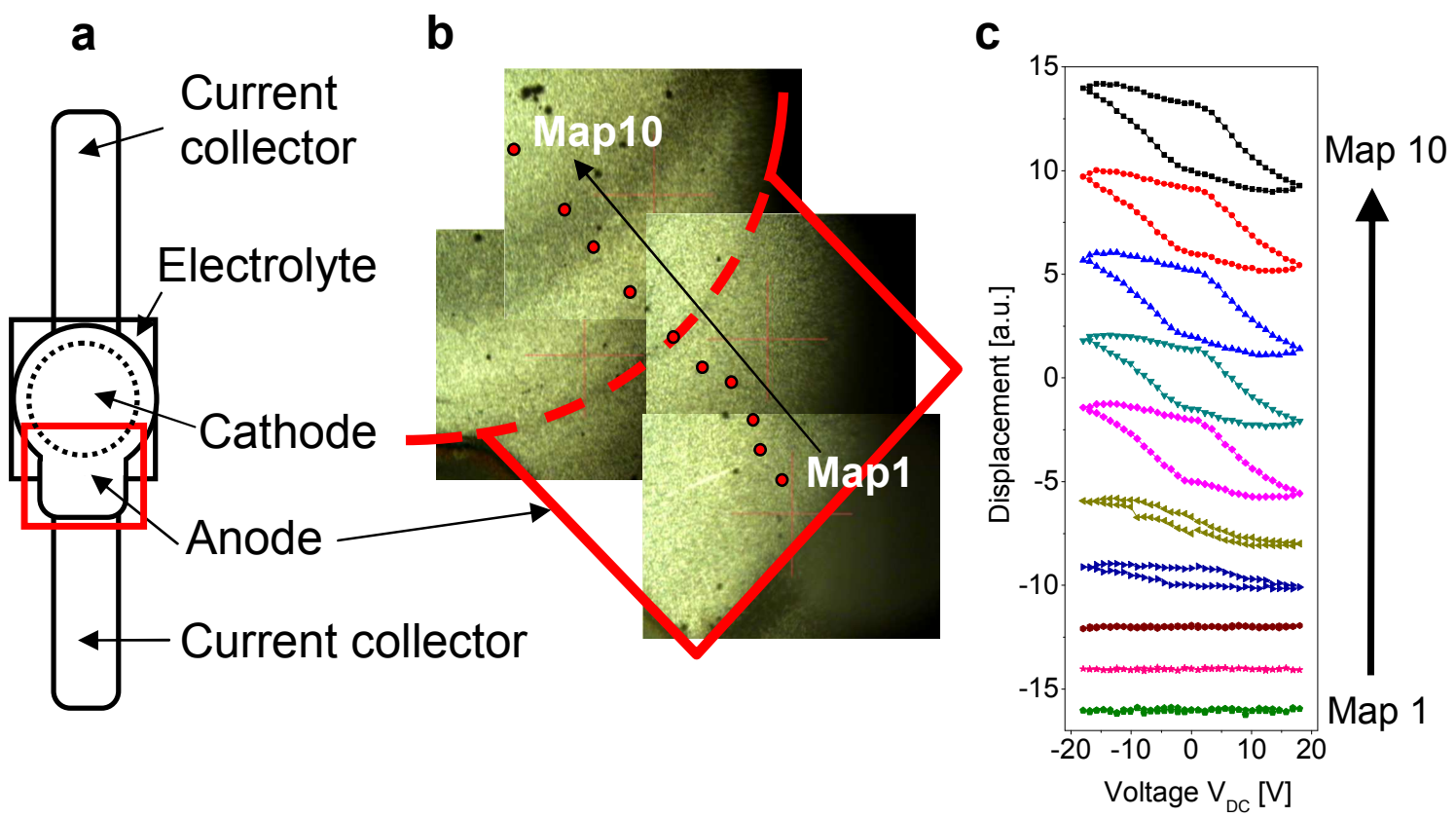

FIG. 2. (a) The layout of the battery device, illustrating the relative position of $\mathrm{LiCoO}_{2}$ cathode, LiPON electrolyte, and Si anode layers. (b) The combination of the optical micrographs of the battery surface acquired through an AFM digital camera system, illustrating the location of measurement locations and the outer layer of the anode. (c) Measured responses (shifted along the yaxis for better visibility) illustrating the lack of electromechanical activity on a bare anode surface and strong hysteretic responses in the regions where underlying LiPON and cathode materials are present. 